\title{
Missed Diagnosis of Liver Cirrhosis Leads to Disparities in Care for Older Patients
}

\author{
Debra Guss $^{\mathrm{a}, \mathrm{b}}$, Jagannath Sherigar ${ }^{\mathrm{a}}$, Smruti R. Mohanty ${ }^{\mathrm{a}}$
}

\begin{abstract}
Background: Cirrhosis of the liver is often not recognized until late in the disease state, when patients decompensate or develop hepatocellular carcinoma (HCC). This inquiry considered factors associated with undiagnosed cirrhosis.
\end{abstract}

Methods: Patients with undiagnosed cirrhosis were compared to patients with known diagnosis of cirrhosis, to evaluate the differences between these two groups. The study population is patients with confirmed diagnosis of HCC, stratified into patients with known diagnosis of cirrhosis $(\mathrm{n}=36)$ and patients without the known diagnosis of cirrhosis who have features of cirrhosis $(n=36)$.

Results: There was no significant difference in insurance, gender, race, etiology of liver disease, presence of splenomegaly, model for end stage liver disease (MELD) score, fibrosis-4 index (FIB-4) or aspartate aminotransferase (AST) to platelet ratio index (APRI) scores between groups. However, the strongest predictor of the diagnosis of cirrhosis was age, with older patients being less likely to be diagnosed with cirrhosis (OR: 0.924, $\mathrm{P}=0.012$ ). Furthermore, tumor size in patients without known cirrhosis was larger than those diagnosed with cirrhosis (median: $4.9 \mathrm{~cm}$ versus 3.5 $\mathrm{cm}, \mathrm{P}=0.015$ ). Of note, $50 \%$ of cases with cirrhosis were undiagnosed.

Conclusion: Older age was the most significant predictor of the missed diagnosis of liver cirrhosis. This led to a larger tumor size at diagnosis, which may imply worse prognosis in these patients. Further evaluation of health disparities related to older age and outcomes of older patients with liver cirrhosis should guide the development of guidelines to prevent the missed diagnosis of cirrhosis.

Keywords: Hepatocellular carcinoma; Health care disparities; Late diagnosis

\section{Manuscript submitted July 31, 2018, accepted September 3, 2018}

${ }^{a}$ New York Presbyterian-Brooklyn Methodist Hospital, Department of Gastroenterology and Hepatobiliary Diseases, Brooklyn, NY 11215, USA

${ }^{b}$ Corresponding Author: Debra Guss, New York Presbyterian-Brooklyn Methodist Hospital, 506 6th Street, Buckley 312, Brooklyn, NY 11215, USA. Email: Dag9132@nyp.org

doi: https://doi.org/10.14740/gr1074w

\section{Introduction}

Cirrhosis of the liver is characterized by chronic progressive liver injury leading to fibrosis and nodularity. Ultimately, cirrhosis progresses to a state of decreased liver functioning and represents the end stage of chronic liver disease, with or without complications associated with cirrhosis. The prevalence of cirrhosis of the liver has nearly doubled in the USA from 2001 to 2013 , equaling nearly $0.3 \%$ of the population, mostly due to hepatitis C infection [1-3]. Cirrhosis of the liver is often not recognized until late in the disease state, due to the natural history of cirrhosis in which a long, compensated, asymptomatic phase is followed by progressively symptomatic decompensation phase. The decompensation phase is defined by the development of complications such as ascites, hepatic encephalopathy and bleeding esophageal varices [1, 4-5]. Mean survival of patients with compensated cirrhosis is $10-12$ years, with mortality occurring when patients pass into the decompensated phase of cirrhosis [5]. Cirrhosis is also a leading cause of hospitalizations with high costs, partly due to the late recognition of disease. If cirrhosis was diagnosed before the development of complications, providers would often be able to prevent decompensation by treating the underlying disease before it created a health crisis [6].

One of the complications of cirrhosis is the development of hepatocellular carcinoma (HCC). HCC is the most common primary cancer of the liver and is the fifth most prevalent cancer worldwide [7]. The major risk factor for the development of HCC is cirrhosis of the liver, with cirrhosis found in more than $80-90 \%$ of liver cancer patients [8,9]. HCC incidence has increased 2.5-fold, and HCC mortality has tripled since 2001 [2]. The 5-year cumulative risk for development of HCC is $5-30 \%$ in patients with cirrhosis [9]. The key to survival of $\mathrm{HCC}$ is early detection of the tumor, through surveillance of patients with cirrhosis of the liver by imaging modalities such as ultrasound, CT scan or MRI of the abdomen $[10,11]$. When $\mathrm{HCC}$ is detected early, it can achieve 5-year patient survival rates near to $50-70 \%$ with resection and/or transplantation versus 3-year survival of $8 \%$ if there are later signs of disease [12].

Surveillance of all patients with cirrhosis for HCC using ultrasound every 6 months is a cost-effective intervention to reduce mortality and improve survival through early detection $[10,11]$. In a well-cited randomized control study $(n=18,816)$, patients who had surveillance for HCC using ultrasound of the liver had a $37 \%$ decrease in mortality [13]. However, it 


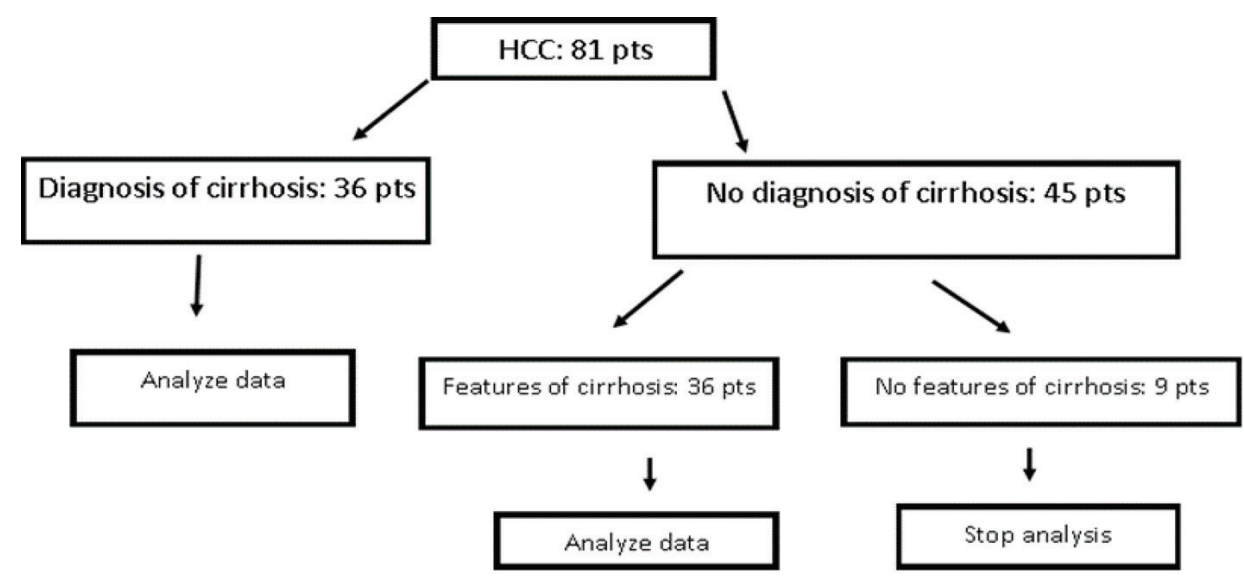

Figure 1. Patients inclusion and exclusion in analysis (pts: patients).

is widely known that adherence to these recommendations is poor, with HCC surveillance rates averaging less than 50\% [7, $14,15,16]$. Under-recognition of cirrhosis has been identified as one cause of the low rate of HCC surveillance $[17,18]$.

Thus, this inquiry will consider the question of which factors are associated with under-diagnosis or undiagnosed cirrhosis in patients with features of liver cirrhosis. In our study, patients with features of cirrhosis who do not have a diagnosis of cirrhosis will be compared to patients with known diagnosis of cirrhosis, to evaluate differences between the two groups, including age, race, gender, insurance type, model for end stage liver disease (MELD) scores and etiology of liver disease. In this study, we included all patients with features of advanced fibrosis or cirrhosis as having features of cirrhosis; this included patients with imaging indicative of cirrhosis, serum fibrosis (i.e. FibroSure) scores of F3 or F4, or liver biopsy results that showed stage 3 or 4 fibrosis. Ultimately, a greater understanding of the factors associated with undiagnosed cirrhosis will lead to an improvement in recognition of cirrhosis and improved outcomes for patients with liver cirrhosis.

\section{Patients and Methods}

\section{Inclusion and exclusion criteria}

This retrospective cross-sectional study involves chart review of patients at an urban medical center in the northeast USA, to determine the differences in the characteristics of patients with a prior diagnosis of cirrhosis versus patients with features of cirrhosis that do not have a diagnosis of cirrhosis. The starting point of this study was patients with $\mathrm{HCC}$, older than age 18 , diagnosis consistent with HCC from 2010 to 2017, and excluded patients with cholangiocarcinoma or other liver cancers (i.e. carcinoid), and hepatitis B (HBV)-related HCC. Diagnosis of HCC was determined by searching the electronic medical record for patients with HCC, ICD 9 code (155.0) or ICD 10 (C22). A thorough record review confirmed the diagnosis of $\mathrm{HCC}$ in patients with imaging results diagnostic of HCC [11], or via tissue sampling through liver biopsy. All patients who meet the inclusion criteria were enrolled in the study.

This study uses HCC as a starting point to find patients with cirrhosis, as $80-90 \%$ of patients with $\mathrm{HCC}$ have cirrhosis. We were then able to work backwards from the diagnosis of $\mathrm{HCC}$, to find patients with cirrhosis who were not previously diagnosed. Patients with HBV-related HCC were excluded, as $\mathrm{HBV}$ is an independent risk factor for the development of HCC, with or without cirrhosis. Thus, it was determined that HCC does not point to a possible diagnosis of cirrhosis in $\mathrm{HBV}$-infected patients.

\section{Study population and research design}

Patients with a diagnosis of HCC from 2010 to 2017 were stratified into two groups. One group had a known diagnosis of cirrhosis, and the second group did not have a known diagnosis of cirrhosis. Patients without a known diagnosis of cirrhosis were further stratified into patients with features of cirrhosis and patients with no features of cirrhosis. Features of cirrhosis were evaluated through an analysis of liver biopsy results, imaging, diagnoses indicative of decompensated liver disease (hepatic encephalopathy, esophageal varices, ascites), laboratory results and serum fibrosis scores (i.e. Fibrotest, Fibrosure). Patient with no features of cirrhosis, or clear evidence that they did not have cirrhosis, were then eliminated from the analysis (Fig. 1).

Clinical and demographic data were collected for all included patients including age, gender, race, insurance, etiology of liver disease, presence of splenomegaly, tumor size at diagnosis, serum fibrosis scores and laboratory results that were used to calculate fibrosis-4 index (FIB-4) and MELD scores (alanine aminotransferase (ALT), aspartate aminotransferase (AST), international normalized ratio (INR), creatinine, sodium, bilirubin, platelet count). Patients with a known diagnosis of cirrhosis were compared to patients with features of cirrhosis without a diagnosis of cirrhosis and statistical analysis was performed (Fig. 1). Mann-Whitney test was used to compare continuous variables between the groups, including tumor size, alpha-fetal protein (AFP), MELD, body mass index (BMI) and AST to platelet ratio index (APRI) scores. The 
groups were further compared using logistical regression and then confirmed with separate chi-square tests for each discrete variable including insurance type, gender, race, etiology of liver disease, splenomegaly, APRI and FIB-4 categories.

\section{Study procedures to determine patient's category}

\section{Patients with cirrhosis diagnosis}

Patients with diagnosis of cirrhosis or mentioned as a problem or elsewhere in the chart during chart review were included in the category of patients diagnosed with cirrhosis. Patients who were diagnosed with cirrhosis of the liver during the same period they were diagnosed with $\mathrm{HCC}$ were coded as no known diagnosis of cirrhosis.

\section{Patients with features of cirrhosis but without a prior diagno-} sis of cirrhosis

Determination of patients as having features of cirrhosis was done through manual chart review of completed diagnostics in patients with $\mathrm{HCC}$ with no previous diagnosis of cirrhosis. First, liver biopsy pathology that mentioned stage III - IV fibrosis was labeled as cirrhosis. By imaging, patients with features suggestive of cirrhosis including nodular liver, shrunken liver, hepatosplenomegaly or mention of cirrhosis were coded as cirrhosis. Serum fibrosis scores indicative of advanced fibrosis or cirrhosis (F3 - F4) were labeled as cirrhosis.

\section{Results}

Two hundred and two patients with HCC diagnosis were included in the initial analysis. Patients were excluded for concomitant diagnosis of HBV $(\mathrm{n}=30)$, liver cancer diagnosis that was not evaluated to be $\mathrm{HCC}$ or indeterminate for HCC $(n=33)$, and lack of information in chart (i.e. during transition to new electronic medical record or patient only coming in for liver biopsy) $(n=58)$, leaving 81 cases for analysis. These 81 cases were further stratified into cases with a known cirrhosis $(\mathrm{n}=36)$ and cases without a known diagnosis of cirrhosis (n $=45$ ). Those cases without known diagnosis of cirrhosis were then analyzed for features of cirrhosis. Nine cases were evaluated to have no characteristics of cirrhosis, and removed from the analysis; the remaining 36 cases with features of cirrhosis, and 36 patients without known diagnosis of cirrhosis (total $n=$ 72) were compared.

Basic descriptive statistics were performed using SPSS to compare the two groups for differences in race, insurance, gender, age, etiology of cirrhosis, presence of splenomegaly (spleen $>11 \mathrm{~cm}$ ), size of tumor, AFP at HCC diagnosis and MELD score. Two validated scores, APRI and FIB-4, are commonly used to determine presence of advanced fibrosis/cirrhosis and were calculated and compared. Mann-Whitney test was used to compare continuous variables between the groups, including tumor size, AFP, MELD, BMI and APRI scores. The groups were further compared using logistical regression and then confirmed with separate chi-square tests for each discrete variable including insurance type, gender, race, etiology of liver disease, splenomegaly, APRI and FIB-4 categories.

\section{Sample description}

Of the 72 patients with HCC and cirrhosis, a full $50 \%(n=36)$ were undiagnosed but had features indicative of cirrhosis assessed through manual chart evaluation of radiological or histological criteria. Age of patients ranged from 44 to 93 years old, with $68.1 \%$ male $(\mathrm{n}=49)$, and varied race with $29.2 \%$ African American $(\mathrm{n}=21), 26.4 \%$ Caucasian $(\mathrm{n}=19), 33.3 \%$ Hispanic $(\mathrm{n}=24), 9.7 \%$ Asian $(\mathrm{n}=7)$ and $1.4 \%$ unknown race $(\mathrm{n}=1)$. Insurance was listed as government insurance (Medicare and/or Medicaid) in $88.9 \%$ of patients $(n=61)$, with the remaining having private insurance $(n=8)$ or none/ unknown insurance $(n=3)$. The etiology of liver disease was overwhelmingly hepatitis $\mathrm{C}$, with $71.8 \%$ of patients with this diagnosis. Though all the included patients were determined to have cirrhosis, FIB-4 scores $>3.25$ indicative of cirrhosis were found in only $79.2 \%$ of patients (Table 1 ).

\section{Findings}

Significantly, $50 \%$ of cirrhotic patients were not previously diagnosed with cirrhosis at the time of HCC presentation. This number of patients with undiagnosed cirrhosis is higher than rates in published literature, which ranged between $23 \%$ and $39 \%$ in patients with HCC [18-20]. The analysis showed no statistically significant difference in insurance, gender, race, etiology of liver disease, the presence of splenomegaly, zip code, MELD, FIB-4 or APRI scores between groups. However, the strongest predictor of the diagnosis of cirrhosis was age, with older-age patients less likely to be diagnosed with cirrhosis (OR: $0.924, \mathrm{P}=0.012$ ). Furthermore, another statistically significant finding was that the tumor size of HCC in patients with undiagnosed cirrhosis was larger (median (Md) $=4.9 \mathrm{~cm})$ than those with known diagnosis of cirrhosis $(\mathrm{Md}=$ $3.5 \mathrm{~cm}, \mathrm{P}=0.015)$.

\section{Discussion}

There are several significant findings in this study. It is noteworthy that $50 \%$ of patients diagnosed with HCC, had features of cirrhosis but had not been previously diagnosed with cirrhosis. Though numerous studies have found a large prevalence of undiagnosed cirrhosis, $23-39 \%$ in patients with HCC, our prevalence of undiagnosed cirrhosis was higher [18, 21]. This may be due to the location within New York City, where patients have many options for care, and seek care at many different institutions. Many of the patients diagnosed with HCC, were being seen for the first time at our institution at the time of HCC diagnosis, and were not engaged in consistent medical care. Patients in this situation may go undiagnosed due to lack 
Table 1. Clinicodemographics Features in HCC Patients With Known Cirrhosis of Features of Cirrhosis but Without a Prior Diagnosis

\begin{tabular}{|c|c|c|c|}
\hline & Known cirrhosis diagnosis $(n=36)$ & No known cirrhosis diagnosis $(n=36)$ & Pvalue \\
\hline Age (mean) & 62.83 & 68.61 & 0.012 \\
\hline Gender (male) & 25 & 24 & 0.880 \\
\hline African American & 9 & 12 & \\
\hline Non-Hispanic white & 11 & 8 & \\
\hline Unknown & 1 & 0 & \\
\hline Insurance & & & 0.952 \\
\hline Medicare & 17 & 17 & \\
\hline Medicaid & 12 & 10 & \\
\hline Etiology & & & 0.513 \\
\hline Cardiac & 0 & 1 & \\
\hline NAFLD & 1 & 1 & \\
\hline Hepatitis C & 22 & 22 & \\
\hline Alcohol & 3 & 5 & \\
\hline Other liver & 2 & 1 & \\
\hline Unknown & 0 & 2 & \\
\hline Alcohol and hepatitis C & 8 & 4 & \\
\hline Presence of splenomegaly & $31 / 36$ & $25 / 36$ & 0.414 \\
\hline MELD score & & & 0.660 \\
\hline$<10$ & 11 & 11 & \\
\hline $10-20$ & 23 & 16 & \\
\hline$>20$ & 2 & 7 & \\
\hline
\end{tabular}

Missed diagnosis of liver cirrhosis leads to disparities in care for older patients.

of care continuity.

Furthermore, the most significant finding is that a missed diagnosis of cirrhosis occurred more commonly in older patients. It is the strongest predictor of missed diagnosis of cirrhosis, and patients in this demographic also had larger HCC tumor size at diagnosis than those patients who had a known diagnosis of cirrhosis. The association between older age and missed cirrhosis diagnosis was also mentioned in prior studies $[20,22]$.

This study replicates a study by Walker et al [18] which considered the same concept of undiagnosed cirrhosis in pa- tients with evidence of cirrhosis and diagnosed with HCC. Walker et al looked at a sample of 1,201 patients from the Veterans Administration (VA) from 2005 to 2011. Similar to our study, they found that patients with undiagnosed cirrhosis were older and had later stage HCC. They also found a larger percentage of undiagnosed patients were African American which we did not find. The VA study only considered patients who received consistent care within the VA, and $92.6 \%$ of these patients had seen a primary care provider at the VA within the last year. This likely explains our higher rate of patients with undiagnosed cirrhosis (50\% versus $24.6 \%$ ), as our patients 
were less likely to have a usual or consistent source of care. Our population also reflected a different demographic and was more urban $(100 \%$ versus $88 \%)$, more diverse $(26.4 \%$ versus $59.2 \%$ white) and less male (68.1\% versus $99.8 \%)$. It is interesting to find similar findings of high rates of undiagnosed cirrhosis with more missed diagnosis in older patients, demonstrating the applicability of the VA findings to a community setting such as ours.

Late diagnosis of cirrhosis or undiagnosed cirrhosis is a phenomenon well known to clinicians. There is a paucity of quality studies that directly consider the missed diagnosis of cirrhosis. This conclusion is often mentioned casually in studies that consider the mortality or etiology of cirrhosis [23, 24], studies recommending screening for cirrhosis in the general population [25-27] or in patients with risk factors [28-30], or as a cause of missed surveillance for HCC in patients with cirrhosis $[18,19]$. The findings of all of these studies suggest that diagnosis of cirrhosis is often missed in patients with and without traditional risk factors. In clinical practice, elevation in liver enzymes is often the catalyst for referral for further testing for liver disease. However, it is known that elevation in liver enzymes is an unreliable marker for cirrhosis [31]. Screening for cirrhosis, particularly in patients with risk factors such as viral hepatitis, obesity or alcohol use, should be mandatory. Any patient with risk factors, or who presents with any sign such as lower than normal platelets, enlarged liver or spleen size, or elevations in liver enzymes should be screened for cirrhosis. Older age should not limit these evaluations and older patients should be screened for history of risk factors, such as alcohol, obesity or previously treated hepatitis $\mathrm{C}$. The absence of active risk factors in older age does not mean that older patients did not have risk factors in the past, and are now living with undiagnosed advanced liver fibrosis or cirrhosis.

The limitations of this study include the small sample size, equaling 72 patients. However, this study is meant to show real world experience of undiagnosed cirrhosis, and is not a population study. Despite the small sample size, it represents a balanced mix of races and included patients with varied insurances including Medicare, Medicaid and private insurance. Though this sample included all etiologies of liver disease except HBV infection, this sample was biased towards patients with hepatitis $\mathrm{C}(\mathrm{HCV})$ cirrhosis caused by longstanding, untreated $\mathrm{HCV}$. In the age of highly efficacious, simple HCV treatments, we anticipate that fewer patients will be living for decades without $\mathrm{HCV}$ treatment and will be less likely to develop HCV-related cirrhosis at the rates found in our population. Instead, the bigger problem will likely be an undiagnosed non-alcoholic fatty liver disease (NAFLD)-related cirrhosis and the applicability of our findings to those patients is uncertain. Furthermore, in patients who presented for the first time to our institution at $\mathrm{HCC}$ diagnosis, it is not clear if they were receiving consistent care at another institution, and so assumptions about prior care and diagnosis cannot definitively be made.

In this study, the greatest risk factor for undiagnosed cirrhosis of the liver was older age, pointing to the failure of our health care system to diagnose cirrhosis in this vulnerable demographic. The discussion of whether to screen patients with risk factors for cirrhosis is ongoing. Currently, screening of patients for cirrhosis is not recommended by any major liver society. This study points to the end-point of this indecision. In our study of patients with HCC and cirrhosis, $50 \%$ of patients with cirrhosis were not diagnosed, with a significant underdiagnosis of older patients. Furthermore, this missed cirrhosis diagnoses led to these patients having a larger tumor size at $\mathrm{HCC}$ diagnosis. Larger tumor size of HCC at diagnosis is associated with poor prognosis. If these tumors were found earlier, there might be more options for treatment. We understand that the discussion of whom to screen for cirrhosis, how often and using what methods remains an important and difficult one. The costs associated with universal screening of patients with risk factors such as NAFLD are enormous. Furthermore, with a dearth of inexpensive, non-invasive options to determine the diagnosis of cirrhosis, easy diagnosis of cirrhosis remains elusive. Continued inquiry and study regarding the benefits of screening high-risk patients and patients with signs of fibrosis/ cirrhosis, requires further focused evaluation. The establishment of guidelines to direct primacy care and other providers to screen high-risk patients should be initiated. More studies that consider the risks, benefits and costs associated with the screening and undiagnosed cirrhosis need to be facilitated in larger populations, and continued examination of non-invasive methods of diagnosis of cirrhosis must continue to be explored.

\section{Acknowledgments}

We thank Dr. Katherine Shurpin, Stony Brook School of Nursing.

\section{Financial Disclosure}

Dr. Smruti Mohanty is a consultant/speaker for Gilead, Intercept, Merck and AbbVie. For the remaining authors, none were declared.

\section{Grant Support}

There was no grant or financial support provided for this research.

\section{Abbreviations}

HCC: hepatocellular carcinoma; HCV: hepatitis C virus; NAFLD: non-alcoholic fatty liver disease; VA: Veterans Administration; MELD: model for end stage liver disease score; FIB-4: fibrosis-4 index; APRI: AST to platelet ratio index

\section{References}

1. D’Amico G, Pasta L, Morabito A, D’Amico M, Caltagirone $\mathrm{M}$, Malizia $\mathrm{G}$, Tine $\mathrm{F}$, et al. Competing risks and prognostic stages of cirrhosis: a 25-year inception cohort study of 494 patients. Aliment Pharmacol Ther. 
2014;39(10):1180-1193.

2. Beste LA, Leipertz SL, Green PK, Dominitz JA, Ross D, Ioannou GN. Trends in burden of cirrhosis and hepatocellular carcinoma by underlying liver disease in US veterans, 2001-2013. Gastroenterology. 2015;149(6):14711482 e1475; quiz e1417-1478.

3. Scaglione S, Kliethermes S, Cao G, Shoham D, Durazo R, Luke A, Volk ML. The Epidemiology of Cirrhosis in the United States: A Population-based Study. J Clin Gastroenterol. 2015;49(8):690-696.

4. Asrani SK, Kamath PS. Natural history of cirrhosis. Curr Gastroenterol Rep. 2013;15(2):308.

5. Gomez EV, Bertot LC, Rodriguez YS, Gonzalez AT, Perez YM, Garcia AY. The natural history of HCV-related cirrhosis and its temporal progression across the different clinical stages. Hepatol Int. 2014;8(4):527-539.

6. Volk ML, Tocco RS, Bazick J, Rakoski MO, Lok AS. Hospital readmissions among patients with decompensated cirrhosis. Am J Gastroenterol. 2012;107(2):247-252.

7. El-Serag HB, Alsarraj A, Richardson P, Davila JA, Kramer JR, Durfee J, Kanwal F. Hepatocellular carcinoma screening practices in the Department of Veterans Affairs: findings from a national facility survey. Dig Dis Sci. 2013;58(11):3117-3126.

8. Gomaa AI, Khan SA, Toledano MB, Waked I, TaylorRobinson SD. Hepatocellular carcinoma: epidemiology, risk factors and pathogenesis. World J Gastroenterol. 2008;14(27):4300-4308.

9. El-Serag HB. Hepatocellular carcinoma. N Engl J Med. 2011;365(12):1118-1127.

10. Bruix J, Sherman M, American Association for the Study of Liver D. Management of hepatocellular carcinoma: an update. Hepatology. 2011;53(3):1020-1022.

11. Heimbach JK, Kulik LM, Finn RS, Sirlin CB, Abecassis MM, Roberts LR, Zhu AX, et al. AASLD guidelines for the treatment of hepatocellular carcinoma. Hepatology. 2018;67(1):358-380.

12. Llovet JM, Bustamante J, Castells A, Vilana R, Ayuso Mdel C, Sala M, Bru C, et al. Natural history of untreated nonsurgical hepatocellular carcinoma: rationale for the design and evaluation of therapeutic trials. Hepatology. 1999;29(1):62-67.

13. Zhang BH, Yang BH, Tang ZY. Randomized controlled trial of screening for hepatocellular carcinoma. J Cancer Res Clin Oncol. 2004;130(7):417-422.

14. Davila JA, Henderson L, Kramer JR, Kanwal F, Richardson PA, Duan Z, El-Serag HB. Utilization of surveillance for hepatocellular carcinoma among hepatitis $\mathrm{C}$ virusinfected veterans in the United States. Ann Intern Med. 2011;154(2):85-93.

15. Singal AG, Marrero JA, Yopp A. Screening process failures for hepatocellular carcinoma. J Natl Compr Canc Netw. 2014;12(3):375-382.

16. Guss DA, Mohanty SR. Integrative review: patient and provider factors related to hepatocellular carcinoma surveillance in patients with liver cirrhosis. Translational Cancer Research. 2017;6(2):450-456.

17. Singal AG, Li X, Tiro J, Kandunoori P, Adams-Huet B, Nehra MS, Yopp A. Racial, social, and clinical deter- minants of hepatocellular carcinoma surveillance. Am J Med. 2015;128(1):90 e91-97.

18. Walker M, El-Serag HB, Sada Y, Mittal S, Ying J, Duan $\mathrm{Z}$, Richardson $\mathrm{P}$, et al. Cirrhosis is under-recognised in patients subsequently diagnosed with hepatocellular cancer. Aliment Pharmacol Ther. 2016;43(5):621-630.

19. Singal AG, Yopp AC, Gupta S, Skinner CS, Halm EA, Okolo E, Nehra M, et al. Failure rates in the hepatocellular carcinoma surveillance process. Cancer Prev Res (Phila). 2012;5(9):1124-1130.

20. Fujimoto K, Sawabe M, Sasaki M, Kino K, Arai T. Undiagnosed cirrhosis occurs frequently in the elderly and requires periodic follow ups and medical treatments. Geriatr Gerontol Int. 2008;8(3):198-203.

21. Farvardin S, Patel J, Khambaty M, Yerokun OA, Mok H, Tiro JA, Yopp AC, et al. Patient-reported barriers are associated with lower hepatocellular carcinoma surveillance rates in patients with cirrhosis. Hepatology. 2017;65(3):875-884.

22. Graudal N, Leth P, Marbjerg L, Galloe AM. Characteristics of cirrhosis undiagnosed during life: a comparative analysis of 73 undiagnosed cases and 149 diagnosed cases of cirrhosis, detected in 4929 consecutive autopsies. J Intern Med. 1991;230(2):165-171.

23. Ratib S, Fleming KM, Crooks CJ, Aithal GP, West J. 1 and 5 year survival estimates for people with cirrhosis of the liver in England, 1998-2009: a large population study. J Hepatol. 2014;60(2):282-289.

24. Dam Fialla A, Schaffalitzky de Muckadell OB, Touborg Lassen A. Incidence, etiology and mortality of cirrhosis: a population-based cohort study. Scand J Gastroenterol. 2012;47(6):702-709.

25. Poynard T, Lebray P, Ingiliz P, Varaut A, Varsat B, Ngo Y, Norha $P$, et al. Prevalence of liver fibrosis and risk factors in a general population using non-invasive biomarkers (FibroTest). BMC Gastroenterol. 2010;10:40.

26. Roulot D, Costes JL, Buyck JF, Warzocha U, Gambier N, Czernichow S, Le Clesiau H, et al. Transient elastography as a screening tool for liver fibrosis and cirrhosis in a community-based population aged over 45 years. Gut. 2011;60(7):977-984.

27. Koehler EM, Plompen EP, Schouten JN, Hansen BE, Darwish Murad S, Taimr P, Leebeek FW, et al. Presence of diabetes mellitus and steatosis is associated with liver stiffness in a general population: The Rotterdam study. Hepatology. 2016;63(1):138-147.

28. Kwok R, Choi KC, Wong GL, Zhang Y, Chan HL, Luk AO, Shu SS, et al. Screening diabetic patients for nonalcoholic fatty liver disease with controlled attenuation parameter and liver stiffness measurements: a prospective cohort study. Gut. 2016;65(8):1359-1368.

29. Harman DJ, Ryder SD, James MW, Jelpke M, Ottey DS, Wilkes EA, Card TR, et al. Direct targeting of risk factors significantly increases the detection of liver cirrhosis in primary care: a cross-sectional diagnostic study utilising transient elastography. BMJ Open. 2015;5(4):e007516.

30. Doycheva I, Cui J, Nguyen P, Costa EA, Hooker J, Hofflich $\mathrm{H}$, Bettencourt R, et al. Non-invasive screening of diabetics in primary care for NAFLD and advanced 
fibrosis by MRI and MRE. Aliment Pharmacol Ther. 2016;43(1):83-95.

31. Verma S, Jensen D, Hart J, Mohanty SR. Predictive value of ALT levels for non-alcoholic steatohepatitis (NASH) and advanced fibrosis in non-alcoholic fatty liver disease (NAFLD). Liver Int. 2013;33(9):1398-1405. 